\title{
Adoption of Robotic Liver, Pancreatic and Biliary Surgery in Singapore: A Single Institution Experience with Its First 100 Consecutive Cases
}

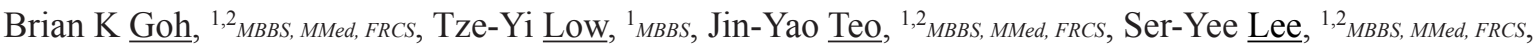
Chung-Yip Chan, ${ }^{1,2}{ }_{M B B S, M D}, F R C S$, Pierce K Chow, ${ }^{1,2}{ }_{M B B S}$, PhD, FRCS, Alexander Y Chung, ${ }^{1,2} M D$, FRCS (London), LPJ $\underline{\text { Ooi }},{ }^{1,2}$ MBBS, MD, FRCS

\begin{abstract}
Introduction: Presently, robotic hepatopancreatobiliary surgery (RHPBS) is increasingly adopted worldwide. This study reports our experience with the first 100 consecutive cases of RHPBS in Singapore.

Methods: Retrospective review of a single-institution prospective database of the first 100 consecutive RHPBS performed over 6 years from February 2013 to February 2019. Eighty-six cases were performed by a single surgeon.

Results: The 100 consecutive cases included 24 isolated liver resections, 48 pancreatic surgeries (including 2 bile duct resections) and 28 biliary surgeries (including 8 with concomitant liver resections). They included 10 major hepatectomies, 15 pancreaticoduodenectomies, 6 radical resections for gallbladder carcinoma and 8 hepaticojejunostomies. The median operation time was 383 minutes, with interquartile range (IQR) of 258 minutes and there were 2 open conversions. The median blood loss was $200 \mathrm{ml}$ (IQR $350 \mathrm{ml}$ ) and 15 patients required intra-operative blood transfusion. There were no post-operative 90-day nor in-hospital mortalities but 5 patients experienced major (> grade 3a) morbidities. The median post-operative stay was 6 days (IQR 5 days) and there were 12 post-operative 30-day readmissions. Comparison between the first 50 and the subsequent 50 patients demonstrated a significant reduction in blood loss, significantly lower proportion of malignant indications, and a decreasing frequency in liver resections performed.

Conclusion: Our experience with the first 100 consecutive cases of RHPBS confirms its feasibility and safety when performed by experienced laparoscopic hepatopancreatobiliary surgeons. It can be performed for even highly complicated major hepatopancreatobiliary surgery with a low open conversion rate.
\end{abstract}

Ann Acad Med Singap 2020;49:742-48

Keywords: Biliary surgery, hepaticojejunostomy, liver resection, pancreas, pancreaticoduodenectomy

\section{Introduction}

The field of hepatopancreatobiliary (HPB) surgery (HPBS), which encompasses major operations on the liver, pancreas or bile ducts, is widely recognised to involve some of the most complicated surgical procedures within the abdominal cavity. Hence, although laparoscopic surgery has rapidly been adopted and become the gold standard for many general abdominal procedures such as cholecystectomies, ${ }^{1,2}$ appendicectomies, ${ }^{3}$ colectomies ${ }^{4}$ adrenalectomies, ${ }^{5}$ gastrectomies ${ }^{6}$ and hysterectomies, ${ }^{7}$ its adoption in the field of HPBS has been relatively slow. ${ }^{8}$ Only over the past decade has the adoption of minimally invasive surgery (MIS) for HPB surgical procedures been increasing rapidly worldwide ${ }^{8,9}$ including Southeast Asia. ${ }^{10-12}$ Even so, the performing of MIS-HPBS remains limited to specialist surgeons in expert centres and a vast majority of major HPB procedures today are still performed via conventional open laparotomy by most surgeons globally.

Several large series of laparoscopic hepatectomies ${ }^{13,14}$ and pancreatectomies ${ }^{15,16}$ have been reported recently in the literature. However, these studies were mainly from

\footnotetext{
${ }^{1}$ Department of Hepatopancreatobiliary and Transplant Surgery, Singapore General Hospital, Singapore

${ }^{2}$ Duke-NUS Medical School, Singapore

Address for Correspondence: Dr Brian KP Goh, Department of Hepatopancreatobiliary and Transplant Surgery, Singapore General Hospital, 20 College Road, Level 5 Academia, Singapore 169856.

Email: bsgkp@hotmail.com
} 
high-volume expert centres, due to the technically demanding nature of most HPBS. ${ }^{10-12}$ Complicated procedures such as major hepatectomies and proximal pancreatectomies are relatively rare and challenging operations to perform via the conventional open approach. Not surprisingly, performing these procedures via conventional laparoscopy has been reported to require a long and steep learning curve, even when done by surgeons highly experienced with the open approach. ${ }^{17,18}$ Hence, the vast majority of HPB surgeons worldwide today still choose to perform these procedures via the open approach despite increasing evidence from randomised controlled trials ${ }^{19,20}$ confirming the advantages of performing these procedures via MIS (such as decreased pain, shorter length of stay and decreased blood loss).

The robotic surgical platform was introduced to overcome many of the limitations of conventional laparoscopy. ${ }^{21}$ One of its main advantages reported was the increased dexterity and stability of its patented Endowrist technology. It allowed surgeons to easily perform more precise and delicate dissection and suturing in extremely tight spaces compared to conventional laparoscopy, useful for HPB procedures such as fine dissection in the hilar region and the performance of difficult anastomoses such as pancreatojejunostomies ${ }^{22}$ and hepaticojejunostomies. ${ }^{23}$ Increasing number of studies have also been published demonstrating the advantages of robotic assistance for hepatectomies ${ }^{24}$ and pancreatectomies. ${ }^{25}$ Other reported advantages of robotic HPB surgery over conventional laparoscopy include the shorter learning curve ${ }^{26}$ and lower open conversion rate $^{27}$ but at the expense of longer operation time and increased cost.

In this present study, we reported our experience with our first 100 consecutive RHPBS. This series represents an update to our previous publication of our first 20 cases. ${ }^{5}$ To our knowledge, this is the largest series of RHPBS in Southeast Asia to date.

\section{Methods}

This was a retrospective review of our single-institution RHPBS database over 6 years between February 2013 and February 2019. We identified the first 100 consecutive patients who underwent RHPBS using the Da Vinci-Si Surgical System (Intuitive Surgical Sunnyvale, California, US). This study was approved by our institutional review board. Peri-operative outcomes of interest such as operative time, total operative blood loss, blood transfusion, post-operative morbidity/mortality and length of stay after surgery were recorded.

Patients were considered for RHPBS when they were determined to be suitable candidates for the minimally invasive surgical approach. The decision for the robotic approach was dependent on multiple factors including individual surgeons' preference and patients' choice after a thorough discussion on the benefits and limitations of the different approaches. Cost and availability of the robot was also a major factor in the decision-making. On average, patients had to pay an additional $\$ \$ 6,000$ for robotic surgery compared to conventional open surgery. However, there were 3 robotic cholecystectomies performed for cholecystitis during an individual surgeon's early learning experience where the patients did not have to pay the additional charges associated with its use. Our surgical technique for RHPBS has been described in detail in our previous studies. ${ }^{21,23,28-30}$ All cases were performed by 4 different principal console surgeons, of which 86 cases were done by one surgeon who subsequently assisted in all the remaining cases.

Operation time was defined as the duration from the time of skin incision to closure. All post-operative morbidity and mortality were recorded and graded using the Clavien-Dindo classification, ${ }^{31}$ up to 30 days after surgery (including readmissions) or within the same hospital stay. Open conversion was defined as any procedure whereby open incision was required to complete the procedure. The exception was for selected hybrid cases such as pancreatoduodenectomy where the reconstruction was performed via a mini-laparotomy incision or Roux-en-Y hepaticojejunostomy, and the construction of the jejuno-jejunostomy was performed extracorporeally. The international study group definition for the classification of post-operative pancreatic fistula was adopted as per our previous studies. ${ }^{12,28}$

In this study we classified pancreaticoduodenectomies, extended pancreatectomies (adjacent organ/vascular resection), major liver resections (resection of $>3$ segments/right anterior segmentectomy/right posterior segmentectomy), ${ }^{10}$ segmentectomy of difficult posterior superior segments, radical resection for gallbladder cancers and hepaticojejunostomies as high-difficulty procedures. The remaining procedures such as distal pancreatectomies and minor liver resections were classified as procedures of intermediate difficulty.

All statistical analyses were conducted using the computer program Statistical Package for Social Sciences for Windows, version 21.0 (SPSS Inc). Univariate analyses were performed using Mann Whitney U, chi-square or Fischer's Exact tests as appropriate. All tests were 2-sided and $P<0.05$ was considered statistically significant.

\section{Results}

One hundred consecutive patients underwent attempted RHPBS during the study period. The operative 
procedures are summarised in Table 1. These included 24 isolated liver resections, 48 pancreatic surgeries (including 2 concomitant bile duct resections) and 28 biliary surgeries (including 8 concomitant liver resections). Also included were 10 major hepatectomies, 15 pancreaticoduodenectomies, 6 radical resections for gallbladder carcinoma and 8 hepaticojejunostomies. There were only 2 open conversions in this series, 1 during a distal pancreatosplenectomy due to tumour extension and 1 for bleeding during resection of a segment 7 hepatic tumour.

Table 1. Details of the robotic operations undergone by the 100 patients

\begin{tabular}{|c|c|c|}
\hline & Operation type & Number \\
\hline \multirow[t]{7}{*}{ Liver resections } & & 24 \\
\hline & Major hepatectomy & $8(2)^{*}$ \\
\hline & Right/extended right hepatectomy & $2(1) *$ \\
\hline & Left hepatectomy & $1(1)$ \\
\hline & Central hepatectomy & 2 \\
\hline & Right posterior sectionectomy & 3 \\
\hline & Minor hepatectomy & $16(6)^{*}$ \\
\hline \multirow[t]{13}{*}{ Pancreatic surgery } & & 48 \\
\hline & Pancreaticoduodenectomy/total pancreatectomy $(\mathrm{n}=1)$ & 15\# \\
\hline & Hybrid (open reconstruction) & 6 \\
\hline & Extended/ vascular resection & 4 \\
\hline & Distal/subtotal pancreatectomy & 25 \\
\hline & Spleen-saving pancreatectomy & 11 \\
\hline & Pancreatosplenectomy & 10 \\
\hline & Extended pancreatosplenectomy & 4 \\
\hline & Others & 8 \\
\hline & Enucleation ( 2 deep lesions close to pancreatic duct) & 3 \\
\hline & Resection of adjacent organ adherent to pancreas \#\# & 3 \\
\hline & Completion pancreatosplenectomy for recurrent tumour (after previous open whipples) & 1 \\
\hline & Lateral pancreatojejunostomy & 1 \\
\hline \multirow[t]{18}{*}{ Biliary surgery } & & 28 \\
\hline & Mirizzi syndrome & 6 \\
\hline & Cholecystectomy & 2 \\
\hline & Subtotal cholecystectomy, bile duct exploration and cholecystoplasty & $4^{* *}$ \\
\hline & Cholecystectomy for severe cholecystitis & 3 \\
\hline & Cholecystectomy and transcholedochal bile duct exploration after failed ERCP & 2 \\
\hline & Cholecystectomy with resection/repair of cholecystoduodenal fistula & 2 \\
\hline & Choledochectomy, bile duct exploration and Roux-en-Y HJ for primary choledocholithiasis & 1 \\
\hline & Triple bypass (Roux-en-Y HJ) with bile duct exploration for ampullary cancer & 1 \\
\hline & Choledochectomy and Roux-en-Y HJ for choledochal cyst & 3 \\
\hline & Choledochectomy and Roux-en-Y HJ (aberrant anatomy with 4-duct) benign stricture & 1 \\
\hline & Choledochectomy and Roux-en-Y HJ for benign stricture & 1 \\
\hline & Left hepatectomy, choledochotomy and removal stone for benign stricture & $1 *$ \\
\hline & Gallbladder cancer & 6* \\
\hline & Radical cholecystectomy with hilar LN clearance & 3 \\
\hline & Segment $4 b / 5$ resection with hilar $L N$ clearance & 2 \\
\hline & Right hepatectomy/ caudate lobe resection, radical choledochectomy and Roux-en-Y HJ & 1 \\
\hline & Resection of hilar LN 1 for recurrent colorectal metastases (2 previous liver resections) & 1 \\
\hline
\end{tabular}

ERCP: endoscopic retrograde cholangiopancreatography; HJ: hepaticojejunostomy; LN: lymph node

* 8 biliary procedures with concomitant liver resections: 6 minor resections and 2 major liver resections

** 1 concomitant minor liver resection

\# 2 pancreaticoduodenectomies with concomitant radical choledochectomy for mid bile duct cancer included under pancreatic resections \#\# Gastric resection with pancreas, left adrenalectomy with pancreas, retroperitoneal tumour with pancreas 
Of the 15 pancreaticoduodenectomies, there was 1 total pancreatosplenectomy and 6 were performed via a hybrid procedure. Four required portal/superior mesenteric vein resections, of which 3 were wedge resections and 1 being a segmental resection with endto-end anastomosis. Two of the wedge resections were performed totally by minimally invasive procedures.

The patients' baseline characteristics and outcomes are summarised in Table 2. Comparison between the initial 50 versus the subsequent 50 cases demonstrated a significant decrease in blood loss but no significant difference in other key peri-operative outcomes. Overall, there were 15 major ( $>$ grade 2 ) morbidities. These are summarised in Table 3. There were no post-operative 90-day or in-hospital mortality in this series. There were 12 readmissions and these were due to infected collection/grade B pancreatic fistula after distal pancreatectomy in 4 patients, infected collection after liver resection in 1 patient, and reflux cholangitis after hepaticojejunostomy in 1 patient. Six patients who underwent pancreaticoduodenectomy and required readmissions included 2 infected intra-abdominal collections, 1 superficial wound infection, 2 delayed gastric emptying and 1 urinary tract infection.

\section{Discussion}

The first series of robotic hepatectomies and pancreatectomies was reported by Giullianotti et al. in 2003. ${ }^{32}$ Subsequently, several large series from expert centres were reported on robotic pancreatectomies ${ }^{25,33}$ and robotic hepatectomies. ${ }^{34,35}$ RHPBS was adopted to overcome the limitations of conventional laparoscopy

Table 2. Evolution of the clinico-pathological features and outcomes of the 100 patients who underwent robotic hepatopancreatobiliary surgery

\begin{tabular}{|c|c|c|c|c|}
\hline & $\begin{array}{c}\text { Total } \\
(\mathrm{n}=100)\end{array}$ & $\begin{array}{c}\text { Group } 1 \\
(1-50)\end{array}$ & $\begin{array}{c}\text { Group } 2 \\
(51-100)\end{array}$ & $P$-value \\
\hline Median age (IQR), years & $63(17)$ & $61(16)$ & $66(16)$ & 0.161 \\
\hline Male, n (\%) & 43 & $20(40)$ & $23(46)$ & 0.545 \\
\hline ASA score, n (\%) & & & & 0.264 \\
\hline 1 & 13 & $9(18)$ & $4(8)$ & \\
\hline 2 & 65 & $32(64)$ & $33(66)$ & \\
\hline 3 & 22 & $9(18)$ & $13(26)$ & \\
\hline Median BMI (IQR) & $23.8(6.7)$ & $24.2(6.0)$ & $23.2(7.6)$ & 0.463 \\
\hline Median tumour size (IQR) & $21(21)$ & $20(32)$ & $23(20)$ & 0.982 \\
\hline Type of surgery, n (\%) & & & & 0.055 \\
\hline Liver & 24 & $17(34)$ & $7(14)$ & \\
\hline Biliary * & 28 & $11(22)$ & $17(34)$ & \\
\hline Difficulty of procedure (\%) & & & & 0.221 \\
\hline Intermediate & 60 & $33(66)$ & $27(54)$ & \\
\hline High & 40 & $17(34)$ & $23(46)$ & \\
\hline Malignancy, n (\%) & 64 & $37(74)$ & $27(54)$ & 0.037 \\
\hline Median operation time (IQR), min & $383(258)$ & $358(241)$ & $393(366)$ & 0.563 \\
\hline Open conversion, $\mathrm{n}(\%)$ & 2 & $2(4)$ & 0 & 0.153 \\
\hline Median blood loss (IQR), mls & $200(350)$ & $275(650)$ & $125(250)$ & 0.027 \\
\hline Intra-operative blood transfusion, $\mathrm{n}(\%)$ & 15 & $11(22)$ & $4(8)$ & 0.050 \\
\hline Median post-operative stay (IQR), d & $6(5)$ & $6(3)$ & $6(6)$ & 0.222 \\
\hline Major morbidity (> grade II), n (\%) & 15 & $4(8)$ & $11(22)$ & 0.05 \\
\hline 90-day/in-hospital mortality, n (\%) & 0 & 0 & 0 & NA \\
\hline
\end{tabular}

ASA: American Society of Anaesthesiologists; BMI: body mass index; IQR: interquartile range

* 8 biliary procedures with concomitant liver resections 
and to shorten the long and steep learning curve associated with laparoscopic hepatectomies and pancreatectomy. ${ }^{36}$ Nonetheless, despite its many theoretical advantages, RHPBS remains a technically challenging procedure especially during the early learning phase. This has been reported by several investigators even from high volume expert centres in HPBS. In the first 77 patients who underwent RHPBS by a single surgeon from the Carolinas Medical Center, US, the authors reported 24 conversions of which $14(18 \%)$ were open conversions. ${ }^{37}$ Similarly, in another large series of robotic distal pancreatectomies reported from the Memorial Sloan Kettering Cancer Center, US, open conversions were required in 14 out of $37(38 \%)$ procedures. ${ }^{38}$

Nonetheless, more recent studies suggest that RHPBS can be adopted safely with a low open conversion rate, especially when performed by surgeons with prior experience with laparoscopic HPBS. Two studies from Taiwan ${ }^{35}$ and Russia ${ }^{26}$ reported that the learning curve for robotic hepatectomy was shorter than for conventional laparoscopy, and this enabled them to perform more complicated and major resections safely with a low open conversion rate. Similarly, Tsung et al. ${ }^{36}$ demonstrated that robotic assistance allowed them to complete more hepatectomies via totally minimally invasive approach without the need of hand-assistance or open conversion compared to conventional laparoscopy. Daoudi et al. reported that the adoption of robotic distal pancreatectomy resulted in lower conversion rates compared to the laparoscopic approach. ${ }^{27}$

Despite this study being our initial experience with the first 100 cases, our open conversion rate was only $2 \%$ even for highly complex procedures, including major hepatectomies and pancreaticoduodenectomies. Comparatively, several local studies had reported open conversion rates ranging from $8 \%$ to $25 \%$ for cholecystectomies ${ }^{1}$ and bile duct exploration $\mathrm{s}^{39}$ performed via conventional laparoscopy. Our early experience even included pancreatectomies with concomitant vascular resections and hepatectomies with bilio-enteric anastomoses. These good results can be attributed to 2 main reasons. Firstly, we had a great deal of experience with both open and laparoscopic HPB surgeries. During the study period, we had concomitant experience with about 500 conventional laparoscopic liver resections and over 100 major laparoscopic pancreatic surgeries. The strong foundation of open HPB surgery, coupled with advanced laparoscopic skills, was likely a major factor accounting for our results.

Secondly, appropriate patient selection was practised. Surgeons learning RHPBS should select cases that are not too complicated and appropriate to their skill level.
In this study, as we accumulated vast experience with open and laparoscopic HPBS, we started RHPBS with moderately difficult cases such as minor hepatectomies or distal pancreatectomies. As this case experience increased, we quickly progressed to major hepatectomies and pancreaticoduodenectomies. We would caution that the present experience may not be the same for all HPB surgeons embarking on RHPBS, in particular for those with minimal experience with the conventional laparoscopic approach. Although the robotic platform has been reported to be simpler to learn than conventional laparoscopy for the open surgeon, the learning curve of robotic surgery for surgeons already proficient with advanced laparoscopic procedures is even shorter. ${ }^{21,23,40}$ This is because many of the skill sets acquired from conventional laparoscopy such as trocar placement, operative technique, magnified surgical field viewed through a television monitor, surgical manoeuvres and operation room setup are easily transferable with only minor modifications to robotic surgery. Similar to our experience, other investigators with prior experience with laparoscopic surgery have also reported excellent outcomes even during their initial experience with RHPBS.

Presently, in our practice, robotic surgery is utilised as an extension of conventional laparoscopic surgery. ${ }^{21,23,28}$ Hence, in our opinion, RHPBS is not competitive but complementary to conventional laparoscopic HPBS today. It is a useful tool to add to the armamentarium of any surgeon practising minimally invasive HPBS. The main advantage of the robotic platform today is its patented Endowrist technology, which allows increased stability, precision and dexterity. This is extremely useful in HPBS especially when performing fine dissection in the hilar region and when constructing complicated anastomoses such as small bilio-enteric and pancreato-enteric anastomoses. ${ }^{23,28,30}$ Robotic assistance expands the capability of the surgeon, enabling him/her to successfully perform more complicated procedures via the minimally invasive approach that would otherwise have to be performed via open surgery. Earlier studies from our centre had suggested that robotic surgery would be especially useful in HPBS such as when performing the complicated reconstructions required for bilio-enteric anastomoses ${ }^{23}$ or pancreatoenteric anastomoses, $, 30,40$ and the complicated dissections needed for spleen-saving pancreatectomies and extended pancreatectomies. ${ }^{11,28}$ At present, we cannot advocate the routine use of RHPBS as a replacement over conventional laparoscopy for all HPB procedures due to the increased cost and limitation of resources.

Nonetheless, it is also important to note that in our experience, the advantages of robotic assistance may be 
Table 3. Details of the major ( $>$ grade 2 ) complications occurring in the 15 patients

\begin{tabular}{|c|c|c|}
\hline Major morbidity type & Grade & Number \\
\hline $\begin{array}{l}\text { Grade B pancreatic fistula ( } 2 \text { distal pancreatectomies, } 2 \text { enucleations and } 1 \text { pancreatoduodenectomy } \\
\text { requiring percutaneous drainage) }\end{array}$ & $3 a$ & 5 \\
\hline Delayed gastric emptying after pancreaticoduodenectomy requiring naso-jejunal tube placement & $3 \mathrm{a}$ & 3 \\
\hline $\begin{array}{l}\text { Infected intra-abdominal collection after right hepatectomy and radical choledochectomy with HJ requiring } \\
\text { percutaneous drainage }\end{array}$ & $3 a$ & 1 \\
\hline Upper gastrointestinal bleed after pancreaticoduodenectomy requiring gastroscopy and clipping & $3 a$ & 1 \\
\hline Port site hernia after distal pancreatectomy requiring reoperation & $3 b$ & 1 \\
\hline Internal hernia after pancreaticoduodenectomy requiring reoperation & $3 b$ & 1 \\
\hline HJ leak requiring reoperation & $3 b$ & 1 \\
\hline Extracorporeal created jejunojejunostomy for Roux limb leak requiring reoperation & $3 b$ & 1 \\
\hline Massive pulmonary embolism with severe hypotension & 4 & 1 \\
\hline
\end{tabular}

less apparent for minimally invasive liver resections. ${ }^{10,21,29}$ This is because the current surgical instruments commonly used for liver parenchyma transection such as the Cavitron Ultrasonic Surgical Aspirator (CUSA) are not yet available via the robotic platform, whereas the presently available robotic harmonic scalpel is a non-articulating instrument that mitigates many of the advantages of the robotic platform over conventional laparoscopy. Notably in our practice, the proportion of robotic assistance for liver resections has progressively decreased from $34 \%$ to $14 \%$. There was a corresponding increase in the use of robotic assistance for complicated pancreatobiliary procedures, including inflammatory/ infectious conditions such as complicated gallstone diseases and strictures. This may have partly accounted for our observations that there was a significant reduction in the blood loss and lower proportion of malignancies between group 1 and group 2 (Table 2). It is important to highlight that the decrease in the use of robotic assistance for liver resections is a potential confounding factor for the lower blood loss observed in group 2 . The increasing proportion of pancreatobiliary surgeries performed in group 2 also likely accounted for the nonsignificant higher morbidity rate observed in this group as pancreatobiliary surgeries are known to be associated with higher morbidity rates compared to liver resections.

Today, the main obstacles to the widespread adoption of RHPBS are the increased cost and limited accessibility for surgeons. ${ }^{21}$ Moreover, with the lack of high-level evidence of its superiority over conventional laparoscopy, ${ }^{24}$ justification for its routine use remains difficult in many countries. As most surgeons will not have unlimited access to robotic technology, resulting in lack of familiarity with the system, this coupled with the steep learning curve of MIS-HPBS has resulted in only a relatively small number of surgeons performing RHPBS globally. ${ }^{23}$ However, it is important to note that over the next few years, many new robotic platforms are coming to the market, which would likely result in a decrease in cost and increase in accessibility. It is also important to add that the role of robotic surgery may be especially important for smaller countries such as Singapore, whereby for most surgeons, individual surgeon case volume for many complicated HPBS may not be high. Hence, a shorter learning curve associated with robotic assistance will be beneficial for more surgeons in the country to embark on MIS-HPBS safely.

\section{Conclusion}

Our experience with the first 100 consecutive cases of RHPBS confirms its feasibility and safety when performed by experienced laparoscopic HPB surgeons. It can be performed for even highly complicated HPB procedures with a low open conversion rate. In our practice, its use complemented and did not not replace conventional laparoscopy, expanding our indications for MIS-HPBS.

\section{REFERENCES}

1. Lim KR, Ibrahim S, Tan NC, et al. Risk factors for conversion to open surgery in patients with acute cholecystitis undergoing interval cholecystectomy. Ann Acad Med Singap 2007;36:631-5.

2. Pucher PH, Brunt LM, Davies N, et al. Outcomes trends and safety measures after 30 years of laparoscopic cholecystectomy: a systematic review and pooled data analysis. Surg Endosc 2018;32:2175-83.

3. Tan WJ, Pek W, Kabir T, et al. Clinical outcome and cost comparison between laparoscopic and open appendicectomy. Ann Acad Med Singap 2014;43:464-8. 
4. Baloyiannis I, Perivoliotis K, Ntellas P, et al. Comparing the safety, efficacy and oncological outcomes of laparoscopic and open colectomy in transverse colon cancer: a meta-analysis. Int J Colorectal Dis 2020;35:373-86.

5. Canniere L, Michel LA. Adrenal and renal surgery by the laparoscopic and/or retroperitoneoscopic approach. Ann Acad Med Singap 1997;26:336-43.

6. $\mathrm{Ng} \mathrm{HJ}, \mathrm{Kim} \mathrm{G}$, Chew CA, et al. Is laparoscopic sleeve gastrectomy for Asian super obese a safe and effective procedure? Ann Acad Med Singap 2018;47:177-84.

7. Ng JS, Fong YF, Tong PS, et al. Gynaecologic robot-assisted cancer and endoscopic surgery (GRACES) in a tertiary referral centre. Ann Acad Med Singap 2011;40:208-12.

8. Ciria R, Cherqui D, Geller DA, et al. Comparative short-term benefits of laparoscopic liver resection: 9000 cases and climbing. Ann Surg 2016;263:761-77.

9. Vollmer CM, Asbun HJ, Barkun J, et al. Proceedings of the first international state-of-the-art conference on minimally-invasive pancreatic resection (MIPR). HPB 2017;19:171-7.

10. Goh BK, Lee SY, Teo JY, et al. Changing trends and outcomes associated with the adoption of minimally invasive hepatectomy: contemporary single-institution experience with 400 consecutive resections. Surg Endosc 2018;32:4658-65.

11. Goh BK, Teo RY. Current status of laparoscopic and robotic pancreatic surgery and its adoption in Singapore. Ann Acad Med Singap 2020;49:377-83.

12. Goh BK, Low TY, Koh YX, et al. Changing trends and outcomes associated with the adoption of minimally invasive pancreatic surgeries: a single institution experience with 150 consecutive procedures in Southeast Asia. J Minim Access Surg 2020;16:404-10.

13. Aldrighetti L, Cipriani F, Fiorentini G, et al. A stepwise learning curve to define the standard for technical improvement in laparoscopic liver resections: complexity-based analysis in 1032 procedures. Updates Surg 2019;71:273-83.

14. Cai X, Duan L, Wang Y, et al. Laparoscopic hepatectomy by curettage and aspiration: a report of 855 cases. Surg Endosc 2016;30:2904-13.

15. Hong S, Song KB, Madkhalo A, et al. Robotic versus laparoscopic distal pancreatectomy for left-sided pancreatic tumors: a single surgeon's experience of 228 consecutive cases. Surg Endosc 2020;34:2465-73.

16. Song KB, Kim SC, Lee W, et al. Laparoscopic pancreaticoduodenectomy for periampullary tumors: lessons learned from 500 consecutive patients in a single centre. Surg Endosc 2020;34:1343-52.

17. Goh BK, Prieto M, Syn N, et al. Critical appraisal of the learning curve of minimally invasive hepatectomy: experience with the first 200 cases of a Southeast Asian early adopted. ANZ J Surg 2020;90:1092-8.

18. Halls M, Alseidi A, Berardi G, et al. A comparison of the learning curves of laparoscopic liver surgeons in differing stages of the IDEAL paradigm of surgical innovation: standing on the shoulders of pioneers. Ann Surg 2019;269:221-8.

19. Fretland AA, Dagenborg VJ, Bjornelv GM, et al. Laparoscopic versus open resection for colorectal liver metastases: The OSLO COMET randomized controlled trial. Ann Surg 2018;267:199-207.

20. Poves I, Burdio F, Morato O, et al. Comparison of perioperative outcomes between laparoscopic and open approach for pancreatoduodenectomy: the PADULAP randomized controlled trial. Ann Surg 2018;268:731-9.

21. Goh BK, Lee SY, Chan CY, et al. Early experience with robot-assisted laparoscopic hepatobiliary and pancreatic surgery in Singapore: single-institution experience with 20 consecutive patients. Singapore Med J 2018;59:133.

22. Watkins AA, Kent TS, Gooding WE, et al. Multicenter outcomes of robotic reconstruction during the early learning curve for minimallyinvasive pancreaticoduodenectomy. HPB 2018;20:155-65.

23. Goh BK, Low TY, Teo JY, et al. Initial single institution experience with robotic biliary surgery and bilio-enteric anastomosis in Southeast Asia. ANZ J Surg 2019;89:E142-46.

24. Wong DJ. Wong MJ, Choi GH, et al. Systematic review and meta-analysis of robotic versus open hepatectomy. ANZ J Surg 2019;89:165-70.

25. Shi Y, Wang W, Qiu W, et al. Learning curve from 450 cases of robot-assisted pancreaticoduodenectomy in a high-volume pancreatic center: optimization of operative procedure and a retrospective study. Ann Surg 2020 (in press).

26. Efanov M, Alikhanov R, Tsvirkun V, et al. Comparative analysis of learning curve in complex robot-assisted and laparoscopic liver resection. HPB 19:818-24.

27. Daouadi M, Zureikat, Zenati, et al. Robot-assisted minimally invasive distal pancreatectomy is superior to the laparoscopic technique. Ann Surg 2013;257:128-32

28. Goh BK, Low TY, Lee SY, et al. Initial experience with robotic pancreatic surgery in Singapore: single institution experience with 30 consecutive cases. ANZ J Surg 2019;89:E137-41.

29. Goh BK, Lee LS, Lee SY, et al. Initial experience with robotic hepatectomy in Singapore: analysis of 48 resections in 43 consecutive patients. ANZ J Surg 2019;89:201-5.

30. Goh BK, Low TY, Kam JH, Lee SY, Chan CY. Initial experience with laparoscopic and robotic surgery for the treatment of periampullary tumors: single institution experience with the first 30 consecutive cases. ANZ J Surg 2019;89:137-41.

31. Dindo D, Demartines N, Clavien PA. Classification of surgical complications. A new proposal with evaluation in a cohort of 6336 patients and result of a survey. Ann Surg 2004;240:205-13.

32. Giulianotti PC, Coratti A, Angelini M, et al. Robotics in general surgery: personal experience in a large community hospital. Arch Surg 2003;138:777-84.

33. Zureikat AM, Beane JD, Zenati MS, et al. 500 Minimally invasive robotic pancreatoduodenectomies: one decade of optimizing performance. Ann Surg 2019 (in press)

34. Chong CN, Lok HT, Fung AK, et al. Robotic versus laparoscopic hepatectomy: application of the difficulty scoring system. Surg Endosc 2019;34:2000-6.

35. Chen PD, Wu CY, Hu RH, et al. Robotic major hepatectomy: Is there a learning curve? Surgery 2017;161:642-49.

36. Tsung A, Geller DA, Sukato DC, et al. Robotic versus laparoscopic hepatectomy: a matched comparison. Ann Surg 2014;259:549-55.

37. Hanna EM, Rozario N, Rupp C, et al. Robotic hepatobiliary and pancreatic surgery: lessons learned and predictors for conversion. Int $\mathrm{J}$ Med Robot 2013;9:152-9.

38. Lee SY, Allen PJ, Sadot E, et al. Distal pancreatectomy: a single institution's experience in open, laparoscopic, and robotic approaches. J Am Coll Surg 2015;220:18-27.

39. Tan KK, Shelat VG, Liau KH, et al. Laparoscopic common bile duct exploration: our first 50 cases. Ann Acad Med Singap 2010;39:136-42.

40. Goh BK, Zeng G, Low TY, et al. Changing trends and outcomes associated with the adoption of minimally-invasive pancreato-biliary surgery: contemporary experience of a 'self-taught' early adopter in Southeast Asia. J Minim Access Surg 2020;16:341-7. 